\title{
Patients with paediatric-onset multiple sclerosis are at higher risk of cognitive impairment in adulthood: An Italian collaborative study
}

\author{
Luis Ruano, Mariana Branco, Emilio Portaccio, Benedetta Goretti, Claudia Niccolai, \\ Francesco Patti, Clara Chisari, Paolo Gallo, Paola Grossi, Angelo Ghezzi, Marco Roscio, \\ Flavia Mattioli, Chiara Stampatori, Marta Simone, Rosa Gemma Viterbo and Maria Pia Amato
}

\section{Abstract}

Background: Patients with paediatric-onset multiple sclerosis (POMS) could be at an increased risk for cognitive impairment (CI), given the potential harmful effects of disease activity in neurodevelopment. However, there is scarce information on their long-term cognitive outcomes.

Objective: To compare the prevalence and profile of CI between adults with a history of POMS and those with classic, adult-onset multiple sclerosis (AOMS).

Methods: Cognitive performance was assessed through the Brief Repeatable Battery (BRB) and the Stroop Test in consecutive patients referred to six Italian MS centres. CI was defined as impairment in $\geqslant 2$ cognitive domains.

Results: In all, 119 patients with POMS and 712 with AOMS were included in this analysis. The prevalence of CI was $48.0 \%$ in AOMS, $44.5 \%$ in POMS; with similar neuropsychological profile between the two groups. However, when adjusting for current age, we found a significantly increased risk for CI (odds ratio $(\mathrm{OR})=1.71 ; p=0.02)$ and for impairment in information processing speed $(\mathrm{OR}=1.86 ; p<0.01)$ in patients with POMS. A higher Expanded Disability Status Scale (EDSS) was also identified in POMS $(p=0.03)$ compared with AOMS patients.

Conclusion: Patients with a history of POMS appear to be at higher risk of physical and cognitive disability than AOMS patients, after correcting for age effects, with particular involvement of information processing speed.

Keywords: Multiple sclerosis, cognitive impairment, paediatric onset, epidemiology

Date received: 6 March 2017; revised: 26 May 2017; accepted: 28 May 2017

\begin{abstract}
Background
Paediatric-onset multiple sclerosis (POMS) occurs in $3 \%-10 \%$ of the whole multiple sclerosis (MS) population $^{1}$ and is usually characterized by a relapsing remitting (RR) course. An increased inflammatory activity seems to be present in paediatric patients, when compared with adult-onset MS (AOMS) patients. ${ }^{2}$
\end{abstract}

Childhood and adolescence represent a critically important period for both brain development and formal academic training. Cognitive impairment (CI) is known to be present in patients with POMS, being consistently reported in approximately one-third of patients, ${ }^{3}$ while in the AOMS population $40 \%-65 \%$ of patients present CI. ${ }^{4}$ The most affected cognitive domains in the paediatric population with MS are similar to those observed in adults, with a predominant involvement of memory, complex attention, information processing speed, executive functions and visual-spatial abilities. ${ }^{3-6}$ Additionally, in POMS subjects, there is accumulating evidence of involvement of linguistic faculties ${ }^{1,6}$ and lower intellectual efficiency in terms of intelligence quotient (IQ), particularly in those with younger age at MS onset. ${ }^{7}$ An early MS disease onset can have a negative influence in school achievements and overall quality of life, ${ }^{8-11}$ but it is unclear if it could lead to an increased risk for CI in later life. ${ }^{3}$ Some studies suggest a negative
Multiple Sclerosis Journal $1-9$

DOI: 10.1177 1352458517717341

(C) The Author(s), 2017. Reprints and permissions: http://www.sagepub.co.uk/ journalsPermissions.nav
Correspondence to: L Ruano

Faculdade de Medicin da Universidade do Porto, Departamento de

Epidemiologia Clinica, Medicina Preditiva e Saúde Pública, Alameda Prof.

Hernâni Monteiro 4200-319 Porto, Portugal. Imruano@gmail.com Luis Ruano EPIUnit, Instituto de Saúde Pública, Universidade do Porto, Porto, Portugal/ Departamento de Epidemiologia Clinica, Medicina Preditiva e Saúde Pública, Faculdade de Medicina, Universidade do Porto, Porto, Portugal/ Neurology Department, Centro Hospitalar de Entre o Douro e Vouga, Santa Maria da Feira, Portugal

Mariana Branco Neurology Department, Centro Hospitalar de Entre o Douro e Vouga, Santa Maria da Feira, Portugal

Emilio Portaccio IRCCS 'Don Carlo Gnocchi', Florence, Italy

Benedetta Gorett Claudia Niccolai Maria Pia Amato

Department NEUROFARBA, Section Neurosciences, University of Florence, Florence, Italy

Francesco Patti Clara Chisari University of Catania, Catania, Italy

Paolo Gallo Paola Grossi University of Padova, Padova, Italy

Angelo Ghezzi Marco Roscio Gallarate Hospital, Varese, Italy

Flavia Mattioli Chiara Stampatori Spedali Civili Brescia, Brescia, Italy 
Marta Simone

Child and Adolescence Neuropsychiatry Unit,

Department of Basic Medical

Sciences, Neuroscience and

Sense Organs, University of

Bari Aldo Moro, Bari, Italy

Rosa Gemma Viterbo

University of Bari Aldo

Moro, Bari, Italy impact of relapses, disease duration and physical disability in the cognitive performance of patients with POMS, ${ }^{8,9,12}$ but these issues remain controversial. ${ }^{10,11}$

There is little information about the long-term cognitive outcome in patients with POMS. Longitudinal studies published to date have relatively short followup periods (1-5 years), and suggest cognitive worsening, with variable rates of decline, although there are patients who may exhibit stable or improved cognitive function over time. ${ }^{3,10,11}$ Some of the heterogeneous results found in the literature are probably related to differences in the demographic and clinical composition of the study samples, as well as to the assessment tools used. The observed improvement in cognitive performance in a subgroup of POMS subjects may be due, in principle, to more efficient brain plasticity and compensatory abilities playing a protective role against CI. ${ }^{3,7}$ Furthermore, there is little data comparing the cognitive performance and profile of CI of adult patients with POMS and AOMS. A single previous study compared the cognitive performance of these two groups, using the Symbol Digit Modalities Test (SDMT), with the results showing a worse test performance in patients with POMS. ${ }^{13}$ A worse performance in language abilities could also be expected, given the more prominent language deficits in the paediatric population. ${ }^{1,6}$

In this cross-sectional, collaborative study, we aimed at comparing the differences in the prevalence and profile of CI in adulthood between adult patients with a history of POMS and patients with classic AOMS. We also explored potential differences between these two groups in terms of other clinical variables, such as disability, fatigue and depression.

\section{Methods}

\section{Study design and setting}

The setting of the study was a nationwide Italian collaborative initiative. During a 6-month period, consecutive patients with MS from six Italian Centres were recruited and underwent a standardized clinical and neuropsychological assessment. The detailed methodology has been previously described. ${ }^{14}$ For this study, a subsample was defined, including the group of adult patients with a history of POMS (diagnosis $\leqslant 18$ years old) and AOMS, confirmed using the 2001 McDonald criteria, ${ }^{15}$ with an RR or secondary progressive (SP) course, defined based on the 1996 Lublin's definition. ${ }^{16}$ The exclusion criteria were current or past neurological disorder other than MS, relapse and/or corticosteroid use within 4 weeks preceding the neuropsychological assessment, major psychiatric illness, history of learning disability before MS onset, alcohol or drug abuse and serious head trauma. Demographical and clinical data including Expanded Disability Status Scale (EDSS) levels and therapeutic information were collected using a common database shared among the participating Centres. In each Centre, a standardized neuropsychological evaluation was performed by a well-experienced neuropsychologist using the Brief Repeatable Battery (BRB) ${ }^{17}$ and the Stroop Test. ${ }^{18}$ The BRB is a widely used and extensively validated for patients with MS. The BRB includes the Selective Reminding Test (SRT), a test that assesses verbal learning by six learning trials and delayed recall of 12 words; the 7/24 Spatial Recall Test (SPART), a test that assesses visuospatial learning by three consecutive trials and a delayed recall of the position of 10 checkers in a checkerboard; the Paced Auditory Serial Addition Test (PASAT), a measure of sustained attention and speed of information processing in which the subject hears single digit numbers and is asked to add each digit to the one preceding it; the SDMT, another measure of sustained attention and speed of information processing in which the subject must associate a pseudorandomized sequence of the symbols with a single digit as quickly as possible, using a key of symbols and digits; and the word list generation (WLG), that explores verbal fluency by asking the subject to produce as many words as possible belonging to a semantic category. ${ }^{17}$ The Stroop Test was selected to complement the BRB in order to provide an additional measure of complex attention and executive function. The neuropsychologists involved in the study participated in a common training session in which test administration and scoring procedures were clarified and agreed upon. Test failure was defined as a score below the 5th or above the 95th percentile, when appropriate, using age, sex and education-adjusted Italian norms. ${ }^{19}$ Impairment in each cognitive domain was defined as failure in a test assessing that domain, namely SRT for Verbal Learning; SPART for Visuospatial Learning; SDMT and PASAT for Information Processing Speed; and WLG and the Stroop test for Executive Function. These theoretical cognitive domains were previously confirmed by principal component analysis in the whole sample. ${ }^{14} \mathrm{CI}$ was defined as impairment in at least two cognitive domains. The fatigue severity scale $(\mathrm{FSS})^{20}$ and the Montgomery and Asberg Depression Scale (MADRS), ${ }^{21}$ while not part of the initial study protocol, were routinely used in several of the study centres, and were also collected during the evaluation to assess fatigue and depression. Data on FSS were available in 96/119 patients with POMS 
and in 502/712 patients with AOMS, MADRS was available in 33/119 and 280/712, respectively. A cutoff of $\geqslant 4$ was used to classify patients as fatigued on the $\mathrm{FSS}^{22}$ and of $\geqslant 20$ to classify patients as moderately or severely depressed on the MADRS. ${ }^{23}$ All the participants provided their written informed consent and the study was approved by the ethics committees of the different institutions.

\section{Statistical analysis}

Group comparisons were performed using Student's $\mathrm{t}$-test for independent samples and the nonparametric Mann-Whitney test or $\chi^{2}$ test, where appropriate. The tests were two-sided, with a significance level of 0.05 . To compare the occurrence of $\mathrm{CI}$ and impairment in each cognitive domain between patients with POMS and AOMS, we calculated crude and adjusted odds ratio (OR) using logistic regression. This analysis was performed adjusting for the effect of age and EDSS that have found to be the independent predictors of $\mathrm{CI}$ in a previous analysis of the complete sample. ${ }^{14}$ Furthermore, we tested the inclusion of disease duration in the logistic regression models adjusted for age, EDSS and POMS, given that a different disease duration is also expected in patients with POMS and AOMS of the same age.

Additionally, and to further clarify the impact on CI of the different disease duration between patients with POMS and AOMS, we performed a complementary analysis of the data by selecting a patient with POMS for each patient with AOMS, best matched for (1) disease duration, (2) age, (3) EDSS and (4) sex, and compared the differences in the prevalence of CI and impairment in each cognitive domain between these matched groups. Statistical analysis was performed using IBM SPSS Statistics for OS X, Version 23.0.

\section{Results}

From the total sample of 1040, 831 adult patients with RR and SP MS were included in this study, 712 with AOMS and 119 with a history of POMS, the excluded participants being clinically isolated syndromes or having a primary progressive course. The refusal rate in the largest study centre (Florence) amounted to $14.5 \%$, other study centres did not keep exact records of refusals; nevertheless, the feedback was that the vast majority of the patients agreed to participate. The main demographic and clinical characteristics of these two groups are shown in Table 1. Median age at onset in patients with POMS was 16.4 years, while it was 29.7 years in patients with AOMS $(p<0.001)$. The education, sex distribution and frequency of current treatment with disease-modifying drugs (DMDs) were also similar between the groups (Table 1).

The prevalence of CI was $48.0 \%$ in AOMS and $44.5 \%$ in POMS $(p=0.49)$. The prevalence of impairment in the different cognitive domains was also similar between the two groups (Table 2), the only significant difference being a higher frequency of verbal learning impairment in patients with AOMS (32.9\% vs $21.8 \%$; $p=0.02)$.

Patients with POMS had longer disease duration, higher EDSS levels and higher number of relapses in the previous year than patients with AOMS (Table 1). When stratifying by age group, the EDSS level remained consistently higher in patients with POMS (Figure 1). The prevalence of patients with FSS $\geqslant 4$ was higher in patients with AOMS $(p=<0.01)$, while the MADRS scores showed no significant differences between groups.

Patients with POMS who exhibited CI in their adulthood $(n=53)$ were older $(p=0.03)$ at the time of examination, had higher EDSS $(p<0.01)$ and longer disease duration $(p=0.02)$ as compared with cognitively preserved POMS patients $(n=66)$. There were no significant differences in sex, education, age at onset and relapses in the previous year (Table 3 ).

In the univariate logistic regression, there were no significant differences in the occurrence of $\mathrm{CI}$ between patients with POMS and AOMS $(\mathrm{OR}=0.87 ; p=0.49$; Table 4). However, adjusting for the effect of age, we found a significantly increased risk for $\mathrm{CI}$ in patients with POMS $(\mathrm{OR}=1.71 ; p=0.02)$. As depicted in Figure 2, the frequency of $\mathrm{CI}$ within each age group was higher in POMS than in AOMS. Performing the same age adjustment for impairment in information processing speed, the OR of POMS increased from 0.96 to 1.86 and the association also became significant $(p<0.01)$. The OR of POMS for impairment in visuospatial learning and executive functions also increased after adjustment although the association remained non-significant ( $p=0.09$ and $p=0.24$, respectively) (Table 4). Contrarily, the increased frequency of impairment in verbal learning in patients with AOMS was no longer significant after adjusting for the effect of age, with a crude OR of $0.57(p=0.02)$ and an adjusted OR of $1.02(p=0.95)$. Adjusting the OR for both age and EDSS, the association between POMS and CI was no longer significant (Table 4). Further adjusting the models for disease duration does not result in a relevant decrease in the OR of POMS for CI (OR adjusted for age and $\mathrm{EDSS}=1.38$; OR 
Table 1. Clinical and demographic characteristics of the study sample.

\begin{tabular}{|c|c|c|c|}
\hline & Adult-onset MS $(n=712)$ & Paediatric-onset MS $(n=119)$ & $p$ value \\
\hline Age, median (IQR), years & $41.9(35.0 ; 49.2)$ & $29.7(24.4 ; 37.9)$ & $<0.001$ \\
\hline Sex (female), $n(\%)$ & $67.7 \%$ & $73.0 \%$ & ns \\
\hline Education, mean (SD), years & $12.1(3.8)$ & $12.8(3.4)$ & ns \\
\hline Age at onset, median (IQR), years & $29.7(24.4 ; 36.4)$ & $16.4(14.6 ; 17.9)$ & $<0.001$ \\
\hline Clinical course (relapsing remitting), $n(\%)$ & $90.3 \%$ & $95.8 \%$ & ns \\
\hline Disease duration, median (IQR), years & $9.2(4.7 ; 16.6)$ & $13.2(8.1 ; 21.5)$ & $<0.01$ \\
\hline Relapses in the previous year, mean (SD) & $0.83(1.0)$ & $1.29(1.1)$ & $<0.001$ \\
\hline EDSS, median (IQR) & $2.0(1.5 ; 4.0)$ & $2.5(1.5 ; 4.0)$ & 0.03 \\
\hline FSS (score $\geqslant 4), n(\%)$ & $66.7 \%$ & $79.5 \%$ & $<0.01$ \\
\hline MADRS (score $\geqslant 20), n(\%)$ & $11.1 \%$ & $3.0 \%$ & ns \\
\hline \multicolumn{4}{|l|}{ Treatment with DMDs, $n(\%)$} \\
\hline No treatment & $31.0 \%$ & $24.8 \%$ & \\
\hline Glatiramer acetate & $9.8 \%$ & $5.5 \%$ & \\
\hline Interferons & $40.0 \%$ & $46.8 \%$ & \\
\hline Natalizumab & $11.2 \%$ & $16.5 \%$ & \\
\hline Fingolimod & $0.6 \%$ & $0.9 \%$ & \\
\hline Immunosuppressant ${ }^{\mathrm{a}}$ & $7.4 \%$ & $5.5 \%$ & ns \\
\hline
\end{tabular}

Table 2. Prevalence and profile of cognitive impairment.

\begin{tabular}{llll}
\hline & $\begin{array}{l}\text { Adult-onset MS } \\
(n=712)\end{array}$ & $\begin{array}{l}\text { Paediatric-onset } \\
\text { MS }(n=119)\end{array}$ & $p$ value \\
\hline Cognitive impairment ( $\geqslant 2$ domains) & $48.0 \%$ & $44.5 \%$ & $\mathrm{~ns}$ \\
Impairment in verbal learning & $32.9 \%$ & $21.8 \%$ & 0.02 \\
Impairment in visuospatial learning & $20.9 \%$ & $22.2 \%$ & $\mathrm{~ns}$ \\
Impairment in information processing speed & $48.5 \%$ & $47.4 \%$ & $\mathrm{~ns}$ \\
Impairment in executive function & $39.8 \%$ & $37.4 \%$ & $\mathrm{~ns}$ \\
Number of impaired domains (impaired patients), mean, $S D$ & $2.6(0.7)$ & $2.5(0.7)$ & $\mathrm{ns}$ \\
Number of impaired domains (all patients), mean, SD & $1.4(1.2)$ & $1.3(1.2)$ & \\
\hline MS: multiple sclerosis; SD: standard deviation; ns: not significant $(p>0.2)$. & & & \\
\hline
\end{tabular}

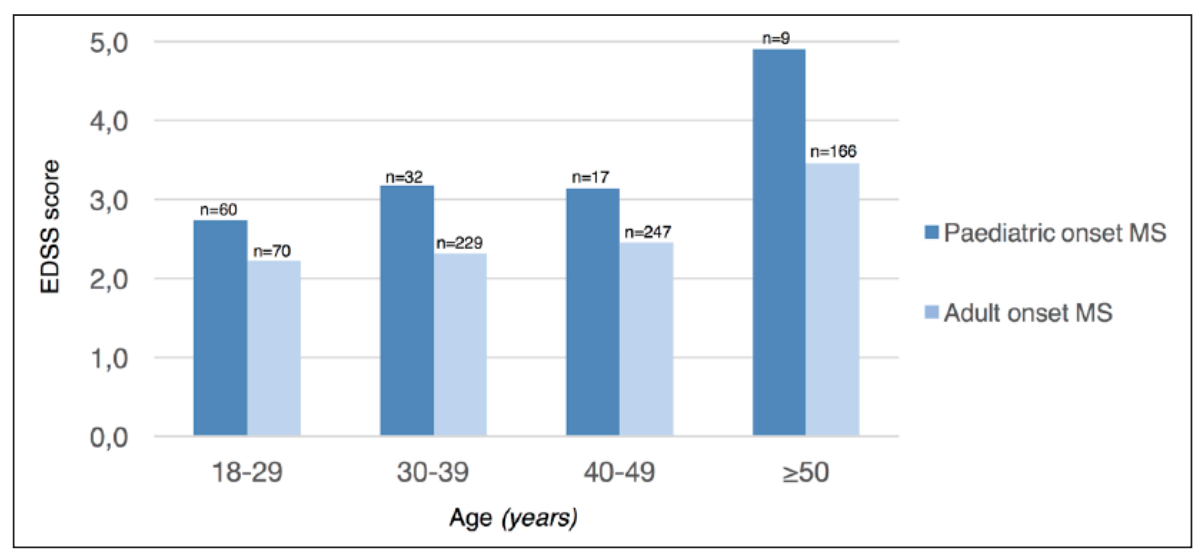

Figure 1. Comparison of the average EDSS score by age group in patients with paediatric- and adult-onset multiple sclerosis. 
Table 3. Comparison of clinical and demographic characteristics between impaired and non-impaired patients with paediatric-onset multiple sclerosis.

\begin{tabular}{|c|c|c|c|}
\hline & $\begin{array}{l}\text { POMS without cognitive impairment } \\
(n=66)\end{array}$ & $\begin{array}{l}\text { POMS with cognitive impairment } \\
(n=53)\end{array}$ & $p$ value \\
\hline Age, median (IQR), years & $27.5(22.7 ; 35.7)$ & $32.3(27.0 ; 39.8)$ & 0.02 \\
\hline Sex (female), $n(\%)$ & $75.0 \%$ & $72.9 \%$ & ns \\
\hline Education, mean (SD), years & $13.2(3.1)$ & $12.5(3.8)$ & ns \\
\hline Age at onset, median (IQR), years & $16.4(14.7 ; 17.9)$ & $16.9(14.2 ; 17.8)$ & ns \\
\hline Disease duration, median (IQR), years & $10.7(6.2 ; 19,3)$ & $15.4(11.0 ; 22.8)$ & 0.03 \\
\hline Relapses in the previous year, mean (SD) & $1.3(1.1)$ & $1.4(1.0)$ & ns \\
\hline EDSS, median (IQR) & $2.25(1.5 ; 3.5)$ & $3.5(2.0 ; 5.0)$ & $<0.01$ \\
\hline \multicolumn{4}{|l|}{ Treatment with DMDs, $n(\%)$} \\
\hline No treatment & $28.3 \%$ & $18.2 \%$ & \\
\hline Glatiramer acetate & $6.7 \%$ & $4.5 \%$ & \\
\hline Interferons & $40.0 \%$ & $52.3 \%$ & \\
\hline Natalizumab & $20.0 \%$ & $13.6 \%$ & \\
\hline Fingolimod & $0 \%$ & $2.7 \%$ & \\
\hline Immunosuppressant ${ }^{\mathrm{a}}$ & $5.0 \%$ & $9.1 \%$ & $\mathrm{~ns}$ \\
\hline
\end{tabular}

Table 4. Odds ratio of paediatric-onset multiple sclerosis for cognitive impairment and impairment in the different cognitive domains.

\begin{tabular}{|c|c|c|c|c|c|c|c|c|}
\hline $\begin{array}{l}\text { Outcome for } \\
\text { POMS }\end{array}$ & $\begin{array}{l}\text { Unadjusted OR } \\
(95 \% \mathrm{CI})\end{array}$ & $\begin{array}{l}p \\
\text { value }\end{array}$ & $\begin{array}{l}\text { OR adj. for age } \\
(95 \% \mathrm{CI})\end{array}$ & $\begin{array}{l}p \\
\text { value }\end{array}$ & $\begin{array}{l}\text { OR adj. for age } \\
\text { and EDSS }(95 \% \\
\text { CI) }\end{array}$ & $\begin{array}{l}p \\
\text { value }\end{array}$ & $\begin{array}{l}\text { OR adj. for age, EDSS } \\
\text { and duration }(95 \% \mathrm{CI})\end{array}$ & $\begin{array}{l}p \\
\text { value }\end{array}$ \\
\hline $\begin{array}{l}\text { Cognitive } \\
\text { impairment }(\geqslant 2 \\
\text { domains) }\end{array}$ & $0.87(0.57 ; 1.03)$ & ns & $1.71(1.07 ; 2.74)$ & 0.02 & $1.38(0.85 ; 2.23)$ & ns & $1.32(0.77 ; 2.23)$ & ns \\
\hline $\begin{array}{l}\text { Impairment in } \\
\text { verbal learning }\end{array}$ & $0.57(0.36 ; 0.91)$ & 0.02 & $1.02(0.62 ; 1.68)$ & ns & $0.82(0.49 ; 1.38)$ & ns & $0.71(0.40 ; 1.26)$ & ns \\
\hline $\begin{array}{l}\text { Impairment in } \\
\text { visuospatial } \\
\text { learning }\end{array}$ & $1.01(0.63 ; 1.63)$ & ns & $1.56(0.93 ; 2.63)$ & ns & $1.32(0.77 ; 2.27)$ & ns & $1.29(0.71 ; 2.35)$ & ns \\
\hline $\begin{array}{l}\text { Impairment in } \\
\text { information } \\
\text { processing speed }\end{array}$ & $0.96(0.65 ; 1.42)$ & ns & $1.86(1.19 ; 2.90)$ & $<0.01$ & $1.53(0.97 ; 2.41)$ & ns & $1.31(0.79 ; 2.17)$ & ns \\
\hline $\begin{array}{l}\text { Impairment in } \\
\text { executive function }\end{array}$ & $0.90(0.59 ; 1.38)$ & ns & $1.32(0.83 ; 2.11)$ & ns & $1.07(0.66 ; 1.74)$ & ns & $1.06(0.61 ; 1.82)$ & ns \\
\hline
\end{tabular}

adjusted for age, EDSS and disease duration=1.32; Table 4). Moreover, when testing this addition of disease duration to the models with age, EDSS and POMS for CI and impairment in the different domains, the association of disease duration with $\mathrm{CI}$ and impairment in different domains is non-significant $(p>0.08)$.

In the analysis of patients with AOMS and POMS matched for disease duration, age, EDSS and sex the prevalence of CI was $28.7 \%$ in POMS and
$44.5 \%$ in AOMS $(p=0.02)$. The prevalence of impairment in each cognitive domain was also higher for patients with POMS although the only difference to reach statistical significance was visuospatial learning (Table 5). Comparing the cognitive test scores and Cohen's $d$ values of the two matched groups, we found significant differences in tests of information processing speed, with moderate effect size for the PASAT test (Cohen's $d$ value $=0.47$ ); Supplementary Table 1$)$. 
Table 5. Comparison of adult and paediatric-onset MS patients, matched for disease duration, age, gender and EDSS.

\begin{tabular}{llll}
\hline & Adult-onset MS $(n=119)$ & Paediatric-onset MS $(n=119)$ & $p$ value \\
\hline Disease duration, median (IQR), years & $12.3(7.3 ; 16.7)$ & $13.2(8.1 ; 21.5)$ & $\mathrm{ns}$ \\
Age, median (IQR), years & $34.9(19.1 ; 38.6)$ & $29.7(24.4 ; 37.9)$ & 0.01 \\
Sex (female), $n(\%)$ & $63.6 \%$ & $73.0 \%$ & $\mathrm{~ns}$ \\
EDSS, median (IQR) & $2.5(1.5 ; 4.0)$ & $2.5(1.5 ; 4.0)$ & $\mathrm{ns}$ \\
Cognitive impairment ( $\geqslant 2$ domains) & $28.7 \%$ & $44.5 \%$ & 0.02 \\
Impairment in verbal learning & $24.2 \%$ & $21.8 \%$ & $\mathrm{~ns}$ \\
Impairment in visuospatial learning & $11.7 \%$ & $21.2 \%$ & 0.04 \\
Impairment in information processing speed & $36.8 \%$ & $47.4 \%$ & $\mathrm{~ns}$ \\
Impairment in executive function & $36.2 \%$ & $37.4 \%$ & $\mathrm{~ns}$ \\
\hline MS: multiple sclerosis; EDSS: Expanded Disability Status Scale; IQR: interquartile range; ns: not significant $(p>0.09)$. & \\
\hline
\end{tabular}

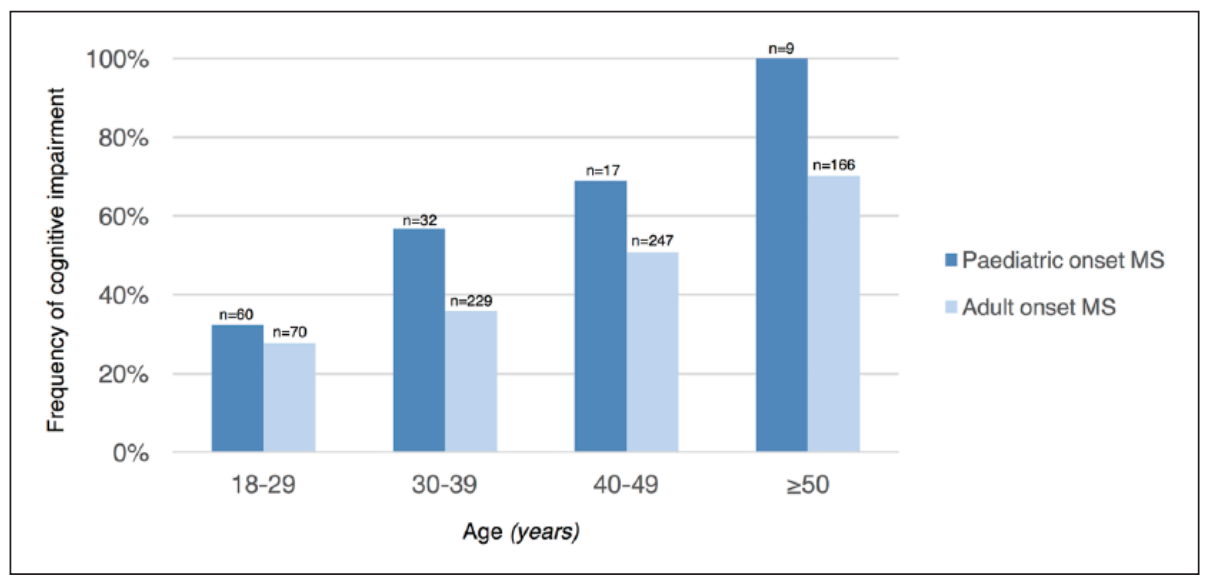

Figure 2. Comparison of the prevalence of cognitive impairment by age group in patients with paediatric-and adult-onset multiple sclerosis.

\section{Discussion}

In this study, we compared the prevalence and profile of CI in a large sample of adult patients with POMS and AOMS, with RR and SP forms, using a standardized neuropsychological battery specifically validated for MS patients.

Our study showed a prevalence of CI of $48.0 \%$ in AOMS and $44.5 \%$ in POMS. These results are in line with what is generally reported in the literature for adult patients with MS $(40 \%-65 \%){ }^{4}$ The prevalence of CI obtained for the adult patients with a history of POMS was higher than that generally found by previous studies in children and adolescents with MS. ${ }^{3}$ This finding reinforces the notion, coming from a few longitudinal studies performed up to date, that, in subjects with a paediatric onset, CI may tend to increase over the years, and those not affected during childhood seem to be at an increased risk to develop cognitive dysfunction later in adulthood, ${ }^{3}$ at an earlier age than their AOMS counterparts. It is important to note that this earlier onset of $\mathrm{CI}$ in patients with POMS could have a negative influence in several aspects of the patient life, namely educational achievements, career making and family planning. Patients with POMS and AOMS presented a similar cognitive profile, with information processing speed being the most commonly affected domain in both groups.

While the crude prevalence of CI was similar between the two groups, after adjusting for the effect of age, we found that patients with POMS had a significantly higher risk for CI than patients with AOMS. This effect was particularly prominent for impairment in information processing speed, with POMS patients having two times increased risk for impairment in this domain than AOMS patients of the same age, and a worse performance in both SDMT and PASAT when compared to matched AOMS patients (Supplementary Table 1). A worse performance of adult patients with POMS in SDMT has been previously described,,$^{13}$ suggesting that information processing speed could 
be disproportionally affected in the long term after an early disease onset. Overall, these findings suggest that adult patients with a history of POMS have worse cognitive outcomes than patients with AOMS of the same age. On the one hand, the onset of MS in a young age, with the cerebral structures and cognitive faculties still in development, could be the main responsible for the increase risk for CI. On the other hand, these results could be explained by the longer disease duration in POMS patients. Since there was a significant association between a paediatric onset of MS and longer disease duration, it was not possible to completely disentangle the independent effect of these two variables in a logistic regression model, also due to the sample size. However, it is interesting to note that further adjusting the model for disease duration does not result in a relevant decrease in the OR for CI of POMS. Furthermore, in the comparison of patients with AOMS and POMS best matched for disease duration, age, EDSS and sex the prevalence of CI was significantly higher POMS than in AOMS ( $44.5 \%$ vs $28.7 \%$; $p=0.02)$. Although the large available sample of AOMS patients, it was not possible to groups were not perfectly match simultaneously for age and disease duration, given that the relative smaller number of younger patients with longer disease duration in the AOMS population when compared with the POMS population, resulting in and older median age for patients with AOMS in this analysis (Table 5). However, given the increased risk of $\mathrm{CI}$ in older patients with MS, any effect of this unbalance in the comparison would be to overestimate the prevalence of CI in AOMS. Consequently, the results from this matched comparison support the notion that the higher risk for $\mathrm{CI}$ in patients with POMS is probably more related to the paediatric onset than to longer disease duration. Additionally, in our previous analysis of the larger sample from which the subsample was selected, we could not find an independent effect of disease duration in the prevalence of $\mathrm{CI}$ in the overall MS population..$^{14}$ Taken as a whole, these findings support the hypothesis that the paediatric onset per se has an important role in determining the cognitive outcomes in adulthood, and that disease duration is probably not the main reason behind the differences in CI performance between adult patients with POMS and AOMS. Additional studies comparing these two groups can be useful to clarify this issue.

Our finding of a similar cognitive profile between the two groups is in accordance to the literature. Previous studies have described language deficits in children with $\mathrm{MS}^{1,3}$ although this topic is controversial. ${ }^{24}$ To explain our results, several considerations should be taken into account. The BRB, used in our study, does not include a specific evaluation of linguistic faculties, which limits our capability to drive any firm conclusion at this regard. In our sample, there were no differences in the two groups in terms of verbal fluency. These results suggest that the verbal fluency deficits described in the paediatric population with MS could be attenuated in adulthood. This may be due to adaptive processes, which are thought to play a role in the improvement of cognition in such cases, ${ }^{10,11}$ and we can hypothesize that formal training through academic education could be related to improved linguistic skills in adulthood. Finally, it is important to note that linguistic deficits are described especially in POMS patients with early disease onset (before 10 years of age), ${ }^{3}$ who represented a minority of subjects in our sample (4.2\%). Long-term follow-up studies of patients with POMS from the diagnosis to adulthood, considering different classes of age at onset, are needed to shed some light on the evolution of different cognitive faculties over the disease course in this special population of patients.

A higher physical disability level was significantly associated with $\mathrm{CI}$ in the previously published analysis of the whole study sample. ${ }^{2}$ In this context, it is important to note that the subgroup of POMS patients with CI also presented higher disability levels although not by a large magnitude, whereas previous studies reached heterogeneous conclusions about the association of physical disability with cognitive dysfunction in patients with POMS. ${ }^{8,10,12}$ It must be noted, however, that the AOMS patients in this sample are significantly older than POMS patients, and when stratifying by age group, the EDSS level remained consistently higher in patients with POMS (Figure 1). In previous prognostic studies, POMS patients appeared to reach irreversible EDSS milestones with a delay of nearly 10 years of disease duration when compared with AOMS patients although these irreversible levels of disability were achieved at a younger age in POMS. ${ }^{25}$

Patients with POMS presented a higher physical disability level than patients with AOMS although they did not exhibit worse results in depression and fatigue scores. Although the fatigue and depression data were incomplete in this sample, the results agree with those of a previous study that compared depression and fatigue between adults of the two groups. ${ }^{13}$ Other studies comparing children with POMS and adult patients with AOMS also showed lower prevalence rates of depression and fatigue in the first group. ${ }^{1}$ This is an interesting finding because higher depression and fatigues scores in adult POMS 
compared to AOMS patients might be expected, due to the reported association with higher physical disability ${ }^{26,27}$ and recent report of an association between psychiatric morbidity and cognitive dysfunction in POMS. ${ }^{28}$ We can hypothesize that these patients could develop better coping strategies, due to living with the diagnosis since early age. Alternatively, the results could also be explained, at least in part, by reduced insight, possibly related with young age at onset and/or higher levels of CI, and a consequent decreased perception of the severity of the disease. Indeed, although a deficit in disability self-awareness has not been extensively researched in patients with MS, some reports have described its presence..$^{29,30}$

In interpreting the study findings, we should consider a few limitations, namely, the cross-sectional design and absence of neuropsychological assessment of the patients at the time of the diagnosis, as well as possible selection of more severe POMS cases referred to specialized MS Centres for adults. Furthermore, it would be interesting to have magnetic resonance imaging (MRI) data to analyse potential differences between the groups and to assess possible correlations of imaging and CI measures.

Despite the above considerations, the results from the study add to previous evidence in the field suggesting that patients with a history of POMS, as compared with their adult-onset counterpart, may be especially vulnerable to the negative consequences of the disease and present worse physical and cognitive outcomes in the long run, particularly regarding impairment in information processing speed. These findings highlight the need for early screening and systematic monitoring of cognitive functioning in the paediatric MS population, aimed at providing prompt counselling and intervention strategies in everyday practice. The development of effective approaches for rehabilitation and prevention of cognitive deterioration in this population remains a priority for future research in this area.

\section{Declaration of Conflicting Interests}

The author(s) declared no potential conflicts of interest with respect to the research, authorship and/or publication of this article.

\section{Funding}

The author(s) disclosed receipt of the following financial support for the research, authorship and/or publication of this article: This study was in part financed through an FISM (Italian Federation of Multiple Sclerosis) research grant.

\section{References}

1. Nunan-Saah J, Paulraj SR, Waubant E, et al. Neuropsychological correlates of multiple sclerosis across the lifespan. Mult Scler 2015; 21: 1355-1364.

2. Waldman A, Ness J, Pohl D, et al. Pediatric multiple sclerosis: Clinical features and outcome. Neurology 2016; 87: S74-S81.

3. Amato MP, Krupp LB, Charvet LE, et al. Pediatric multiple sclerosis: Cognition and mood. Neurology 2016; 87: S82-S87.

4. Amato MP, Zipoli V and Portaccio E. Multiple sclerosis-related cognitive changes: A review of cross-sectional and longitudinal studies. J Neurol Sci 2006; 245: 41-46.

5. Chiaravalloti ND and DeLuca J. Cognitive impairment in multiple sclerosis. Lancet Neurol 2008; 7: 1139-1151.

6. Amato MP, Goretti B, Ghezzi A, et al. Cognitive and psychosocial features of childhood and juvenile MS. Neurology 2008; 70: 1891-1897.

7. Pasto L, Portaccio E, Goretti B, et al. The cognitive reserve theory in the setting of pediatric-onset multiple sclerosis. Mult Scler 2016; 22: 1741-1749.

8. MacAllister WS, Belman AL, Milazzo M, et al. Cognitive functioning in children and adolescents with multiple sclerosis. Neurology 2005; 64: $1422-$ 1425 .

9. Banwell BL and Anderson PE. The cognitive burden of multiple sclerosis in children. Neurology 2005; 64: 891-894.

10. Amato MP, Goretti B, Ghezzi A, et al. Neuropsychological features in childhood and juvenile multiple sclerosis: Five-year follow-up. Neurology 2014; 83: 1432-1438.

11. Amato MP, Goretti B, Ghezzi A, et al. Cognitive and psychosocial features in childhood and juvenile MS: Two-year follow-up. Neurology 2010; 75: 1134 1140.

12. Julian L, Serafin D, Charvet L, et al. Cognitive impairment occurs in children and adolescents with multiple sclerosis: Results from a United States network. J Child Neurol 2013; 28: 102-107.

13. Baruch NF, O’Donnell EH, Glanz BI, et al. Cognitive and patient-reported outcomes in adults with pediatric-onset multiple sclerosis. Mult Scler 2016; 22: $354-361$.

14. Ruano L, Portaccio E, Goretti B, et al. Age and disability drive cognitive impairment in multiple sclerosis across disease subtypes. Mult Scler 2016; 1: 1-10.

15. McDonald WI, Compston A, Edan G, et al. Recommended diagnostic criteria for multiple 
sclerosis: Guidelines from the International Panel on the diagnosis of multiple sclerosis. Ann Neurol 2001; 50: 121-127.

16. Lublin FD and Reingold SC. Defining the clinical course of multiple sclerosis: Results of an international survey. National Multiple Sclerosis Society (USA) Advisory Committee on Clinical Trials of New Agents in Multiple Sclerosis. Neurology 1996; 46: 907-911.

17. Bever CT Jr, Grattan L, Panitch HS, et al. The Brief Repeatable Battery of neuropsychological tests for multiple sclerosis: A preliminary serial study. Mult Scler 1995; 1: 165-169.

18. Stroop JR. Studies of interference in serial verbal reactions. J Exp Psycho 1935; 18: 643-662.

19. Amato MP, Portaccio E, Goretti B, et al. The Rao's Brief Repeatable Battery and Stroop Test: Normative values with age, education and gender corrections in an Italian population. Mult Scler 2006; 12: 787-793.

20. Krupp LB, LaRocca NG, Muir-Nash J, et al. The fatigue severity scale. Application to patients with multiple sclerosis and systemic lupus erythematosus. Arch Neurol 1989; 46: 1121-1123.

21. Montgomery SA and Asberg M. A new depression scale designed to be sensitive to change. $\mathrm{Br} J$ Psychiatry 1979; 134: 382-389.

22. Valko PO, Bassetti CL, Bloch KE, et al. Validation of the fatigue severity scale in a Swiss cohort. Sleep 2008; 31: 1601-1607.
23. Snaith RP, Harrop FM, Newby DA, et al. Grade scores of the Montgomery-Asberg depression and the clinical anxiety scales. Br J Psychiatry 1986; 148: 599-601.

24. Parrish JB, Farooq O and Weinstock-Guttman B. Cognitive deficits in pediatric-onset multiple sclerosis: What does the future hold? Neurodegener Dis Manag 2014; 4: 137-146.

25. Renoux C, Vukusic S, Mikaeloff Y, et al. Natural history of multiple sclerosis with childhood onset. $N$ Engl J Med 2007; 356: 2603-2613.

26. Lynch SG, Kroencke DC and Denney DR. The relationship between disability and depression in multiple sclerosis: The role of uncertainty, coping, and hope. Mult Scler 2001; 7: 411-416.

27. Mattioli F, Bellomi F, Stampatori C, et al. Depression, disability and cognitive impairment in multiple sclerosis: A cross sectional Italian study. Neurol Sci 2011; 32: 825-832.

28. Weisbrot D, Charvet L, Serafin D, et al. Psychiatric diagnoses and cognitive impairment in pediatric multiple sclerosis. Mult Scler 2014; 20: 588-593.

29. Prigatano GP, Hendin BA and Heiserman JE. Denial or unawareness of cognitive deficit associated with multiple sclerosis? A case report. J Clin Exp Neuropsychol 2014; 36: 335-341.

30. Reich E, Arias E, Torres C, et al. Anosognosia and self awareness in multiple sclerosis. J Neurol Sci 2015; 357: e317.
Visit SAGE journals online journals.sagepub.com/ home/msj

(\$) SAGE journals 